\title{
DeepMuCS: A Framework for Mono- \& Co-culture Microscopic Image Analysis: From Generation to Segmentation
}

This paper was downloaded from TechRxiv (https://www.techrxiv.org).

\section{LICENSE}

CC BY 4.0

SUBMISSION DATE / POSTED DATE

$16-02-2022$ / 22-02-2022

\section{CITATION}

Khalid, Nabeel; Koochali, Mohammadmahdi; Rajashekhar, Vikas; Munir, Mohsin; Edlund, Christoffer; Jackson, Timothy; et al. (2022): DeepMuCS: A Framework for Mono- \& Co-culture Microscopic Image Analysis: From Generation to Segmentation. TechRxiv. Preprint.

https://doi.org/10.36227/techrxiv.19181552.v1

$\mathrm{DOI}$

10.36227/techrxiv.19181552.v1 


\title{
DeepMuCS: A Framework for Mono- \& Co-culture Microscopic Image Analysis: From Generation to Segmentation
}

\author{
Nabeel Khalid*, Mohammadmahdi Koochali*, Vikas Rajashekhar*, Mohsin Munir*, Christoffer Edlund ${ }^{\dagger}$, \\ Timothy R Jackson ${ }^{\ddagger}$, Johan Trygg ${ }^{\dagger \S}$, Rickard Sjögren ${ }^{\dagger \S}$, Andreas Dengel*ף ${ }^{*}$, Sheraz Ahmed ${ }^{*}$ \\ ${ }^{*}$ German Research Center for Artificial Intelligence (DFKI) GmbH, Kaiserslautern 67663, Germany \\ firstname.lastname@dfki.de \\ ${ }^{\dagger}$ Sartorius Corporate Research, Sweden \\ ${ }^{\ddagger}$ Sartorius, BioAnalytics, Royston, United Kingdom \\ firstname.lastname@Sartorius.com

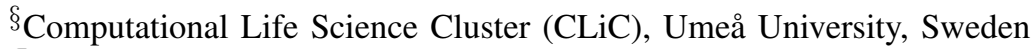 \\ ITechnische Universität Kaiserslautern, Kaiserslautern 67663, Germany
}

\begin{abstract}
Discrimination between cell types in the co-culture environment with multiple cell lines can assist in examining the interaction between different cell populations. Identifying different cell cultures along with segmentation in co-culture is essential for understanding the cellular mechanisms associated with disease states. Extracting the information from the co-culture models can help in quantifying the sub-population response to treatment conditions. In the past, there exists minimal progress related to cell-type aware segmentation in the monoculture and no development whatsoever for the co-culture. The introduction of the LIVECell dataset has provided us with the opportunity to perform experiments for cell-type aware segmentation. However, it is composed of microscopic images in a monoculture environment. In this paper, we have proposed a pipeline for coculture microscopic images data generation, where each image can contain multiple cell cultures. In addition, we have proposed a pipeline for culture-dependent cell segmentation in monoculture and co-culture microscopic images. Based on extensive evaluation, it was revealed that it is possible to achieve good quality cell-type aware segmentation in mono- and co-culture microscopic images.
\end{abstract}

Index Terms-biomedical, healthcare, deep learning, cell segmentation, co-culture

\section{INTRODUCTION}

In drug development, biologists are interested in co-culture models not only because they better replicate the tumor environment in vivo, but it also has a measurable effect on cancer cell response to treatment. In the study [1], prostate cancer cell proliferation is inhibited by several different drugs in monoculture, but to a lesser extent in co-culture with stromal cells. This suggests that monoculture conditions alone are not sufficient to select a suitable treatment for prostate cancer, making co-culture the much more relevant model for drug discovery. There are specific fields in drug discovery such as antibody-drug conjugate (ADCs) development, where the drug is specifically designed to only kill cancer cells. However, there is an important phenomenon known as the "bystander effect" - that is ADCs often still can harm surrounding cells and tissue. Being able to identify the different cell types in a co-culture model will enable us to understand how effective an ADC is at specifically killing cancer cells as well as if it has an impact on other cell types. This insight is critical for designing a drug that has maximum efficacy on cancer while minimizing harm to the rest of the body [2].

Deep learning-based approaches are showing promising results in microscopic image analysis [3] [4] and datasets to train instance segmentation models in multiple cultural environments, such as EVICAN60 [5] and LIVECell [4], are now available to further explore deep learning-based approaches for celltype aware segmentation. In this research, we are using the modified version of LIVECell [4] dataset with eight classes for each cell type instead of one class in the LIVECell dataset. In the cell biology domain, there is no co-culture dataset available. To tackle that issue, we have proposed a pipeline to generate multiple subsets of synthetic co-culture images using the LIVECell dataset. The purpose behind generating the synthetic co-culture images with multiple cell types in a single image is to investigate the ability of the proposed pipeline in the co-culture environment with different experimentation. Compared to multi-class segmentation in natural images [6], segmenting multiple cell types in microscopic images suffer from certain challenges including low contrast as well as irregularly shaped and overlapping cells of different cell types, making segmentation challenging. Unlike normal objects, the different cell types in the microscopic images don't vary a lot with regard to the morphological characteristics. Multiple cell types in the same microscopic image can possess similar properties like low contrast, overlapping cells, and unclear boundaries. In the proposed pipeline, parameters for features extraction and anchor sizes are proposed to detect, segment, and classify different cell cultures with good precision using 
variations of the LIVECell [4] dataset.

We propose a framework for mono- \& co-culture microscopic images analysis. The main contributions of this study are as follows:

- Pipeline to generate co-culture microscopic images with multiple cell cultures in each image.

- An end-to-end pipeline for deep learning based multiple cell type segmentation in monoculture and co-culture environments.

- Extensive evaluation of proposed method using different variations of the LIVECell [4] dataset.

\section{Literature REVIEW}

There are many available studies addressing detection and segmentation of cell and nucleus using traditional computer vision algorithms [7]-[10] and deep learning-based approaches [3]-[5], [11]-[17]. Traditional computer vision approaches use procedures like intensity thresholding, region accumulation, and deformable model fitting [18]. The problem with the traditional computer vision approaches is that they are designed for a specific use case, require manual feature extraction, and lack generalization capacity. Deep Learning (DL) approaches for cell segmentation have opened doors to many applications mostly because they require less domain knowledge and are completely data-driven. Ronneberger et al. (2015) proposed Unet, which won the ISBI 2015 cell tracking and segmentation challenge. The introduction of U-net prompted drastic progress in the field of biomedical research with the development of DeepCell [16] and Usiigaci [17]. Khalid et al. (2021) [3] proposed a pipeline for cell and nucleus segmentation using the EVICAN dataset [5]. Edlund et al. (2021) proposed anchor-free and anchor-based pipelines for cell segmentation on the LIVECell dataset [4].

Unfortunately, there are very few approaches that address the cell-type aware segmentation in a monoculture environment. Khalid et al. (2021) [11] proposed a pipeline for celltype aware instance segmentation in monoculture microscopic images using the EVICAN60 [5]. The author also reported several limitations of the EVICAN60 dataset. When it comes to cell-type aware segmentation in co-culture microscopic images, there is no method or dataset available.

\section{DeepMuCS-Genration: Proposed Co-Culture Microscopic DATA GENERATION PiPELINE}

Fig. 1 provides a system overview of the proposed coculture microscopic data generation pipeline. Synthetic coculture images are generated using the original LIVECell dataset in the steps as follows:

1) Cell instances from different cell cultures are extracted using their segmentation annotations.

2) Backgrounds are generated by initially filtering out the cells from the original images. Subsequently, noise artifacts are extracted from the original images and pasted on the filtered image on a random basis to make the synthetic images realistic.
3) Extracted cell instances from different cell cultures are pasted on the generated background images to obtain the synthetic co-culture images.

The synthetic data is generated according to several rules i.e., the same cell shouldn't appear more than once in an image or in the same position in any other image. A minimum and a maximum number of cell cultures in one image should be 2 and 4 respectively. One image should contain a minimum and maximum of 5 and 20 cell instances in total respectively on a random basis

\section{DeepMuCS-Segmentation: Proposed Cell Segmentation Pipeline for Mono- \& Co-Culture MicRosCOPIC IMAGES}

Fig. 2 provides a system overview of the proposed cell segmentation pipeline for mono- \& co-culture microscopic images. DeepMuCS-Segmentation is based on Feature Pyramid Network [19] with ResNeSt-200 [20] and Deformable Convolution [21]. Cascade Mask R-CNN [13] is used as the prediction head. The proposed pipeline is divided into three components.

\section{A. Feature Extraction}

The purpose of this block is to extract feature maps from the input image at different scales. The feature extraction module of our proposed methodology is composed of Feature Pyramid Network [19] along with ResNeSt-200 [20]. Feature Pyramid Network (FPN) extracts features from the images using a pyramid scheme. It utilizes deep convolutional networks (CNNs) for computing features. FPN combines the low resolution, semantically strong features with high resolution, semantically weak features. It takes a single-scale image as an input and outputs feature maps of proportional size at multiple levels by operating on a bottom-up pathway, top-down pathway, and lateral connections. The bottom-up pathway uses a normal feed-forward $\mathrm{CNN}$ architecture to compute a hierarchy of features consisting of feature maps at various scales. The output of each $\mathrm{CNN}$ layer is used later in the top-down pathway via lateral connections. We are using ResNeSt-200 [20] with deformable convolution [21] as a feed-forward CNN architecture in the bottom-up pathway of our approach. ResNeSt comprises a modular split attention block that allows attention across feature-map groups. These blocks are then stacked according to ResNet-style [22]. We have used deformable convolution [21] instead of conventional convolution in the ResNeSt because it suits best for our application in terms of cell deformity [23]. The output of each convolution layer of ResNeSt is used in the top-down pathway which constructs higher resolution layers from the semantic rich layer. As the final task, the FPN applies a 3x3 convolution operation on each merged map to overcome the aliasing effect after the up sampling to generate the final feature map.

\section{B. Object Region Detection and Groundtruth Matching}

After the extraction of multi-scale features from the backbone network, these features are then passed onto a Regional 


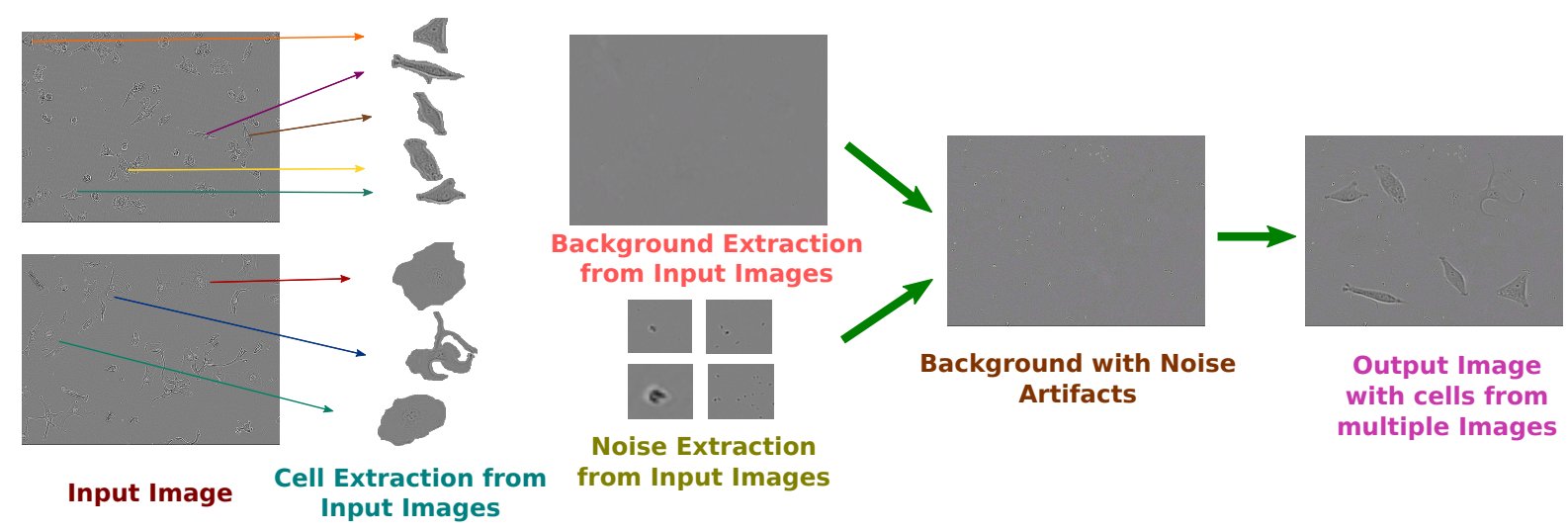

Fig. 1: System overview of DeepMuCS-Genration. Input Images from different cell cultures are passed to the proposed pipeline and the output co-culture images are produced.

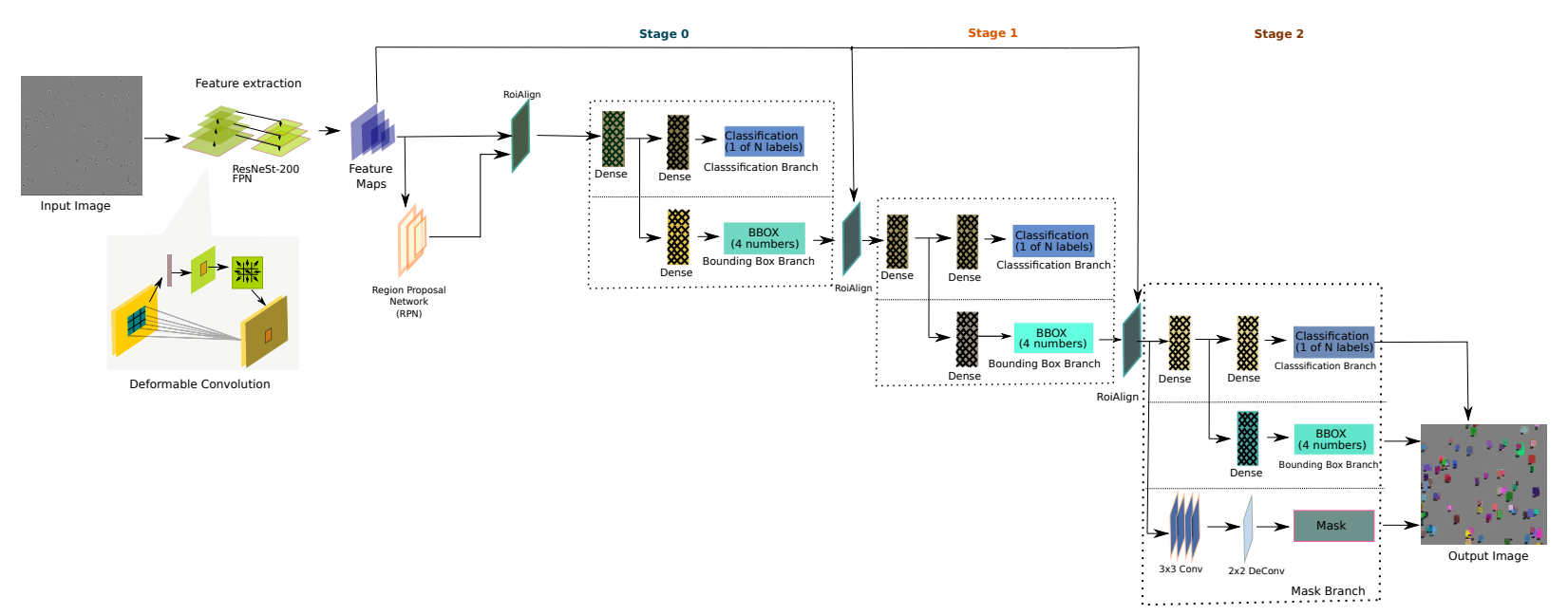

Fig. 2: System overview of DeepMuCS-Segmentation. Input image is passed to the proposed pipeline and the output image with cell-type aware detection and segmentation is produced.

Proposal Network (RPN) [24]. The primary focus of RPN is to detect regions that contain objects and match them to the groundtruth. This process is performed by generating anchor boxes on the input image which are then matched to the groundtruth by taking Intersection over Union (IoU) between anchors and groundtruth. If IoU is larger than the defined threshold of 0.7 , the anchor is linked to one of the groundtruth boxes and assigned to the foreground. If the IoU is greater than 0.3 , it is considered as background and otherwise ignored. The anchor strides and aspect ratio parameter used to detect and segment objects in MS-COCO [6] dataset overlooks most of the small cell instances when transferred to this task. Unlike MS-COCO [6] and other commonly used image datasets, the area of some cells especially BV-2 cell culture in the LIVECell [4] dataset is very small. After extensive experimentation, the anchor sizes and anchor aspect ratios were selected that fit adequately for the task. The details about the anchor parameters are given in Section VII. Now that we have the anchor boxes which are assigned to the foreground having shapes similar to the groundtruth boxes, the next step is anchor deltas calculation which is the distance between groundtruth and anchors. At the final stage of RPN, we choose 3,000 region proposal boxes from the predicted boxes by using non-maximum suppression [13].

\section{Prediction Head}

After the successful generation of proposals, the next block in our pipeline is the prediction head. At the prediction head, we have groundtruth boxes, proposal boxes from RPN, and feature maps from FPN. The job of the prediction head is to predict the class, bounding box, and binary mask for each region of interest. We are using Cascade Mask R-CNN [13] as the prediction head, which is an extension of Cascade RCNN by adding a mask branch to the cascade. Cascade Mask $\mathrm{R}-\mathrm{CNN}$ addresses the problem of making predictions that are more accurate on a pixel level. The architectures like Mask R-CNN [25] usually fail while accurately detecting objects of variable quality and size in an image. This is mainly because the models are trained using a single IoU threshold, which is 0.5 , meaning that the prediction which has over $50 \%$ match with the groundtruth will be regarded as positive samples. This can cause the model to create very bad proposals. To address 
this problem, Cascade Mask R-CNN [13] is used which is a multi-stage network with the IoU threshold increasing for each stage. In the first stage, the outputs of RPN with IoU threshold $<0.5$ are discarded and the positive predictions $(\mathrm{IoU}>0.5)$ are passed onto the next stage. At the second stage, the bounding box predictions from the first stage are regarded as new region proposals. Here the IoU threshold of 0.6 is used to extract positive samples which are then passed onto the final stage, where IoU of 0.7 is used to further refine the output bounding boxes. Similar to Mask R-CNN [25], where the segmentation branch is added in parallel to the classification and bounding box regression. In Cascade Mask $\mathrm{R}-\mathrm{CNN}$, a similar procedure is done, but unlike Mask R-CNN, where we had just one stage, here we have 3 stages. In our methodology, we are adding the segmentation branch at the last stage of the Cascade R-CNN. The box head classifies the object within the ROI and fine-tunes the shape and position of the box. The mask head is composed of a small Fully Convolutional Network (FCN) applied to each ROI, which predicts a segmentation mask in a pixel-to-pixel manner [25] to achieve the task of instance segmentation.

\section{DATASET}

Many datasets are available for cell segmentation [15], [26], [27], but all these datasets either contain images for one cell culture or they don't differentiate between different cell cultures. Only two datasets contain multiple cell lines in the monoculture microscopic images i.e., EVICAN60 [5] and the LIVECell [4]. The EVICAN60 dataset contains classes for 30 cell cultures. EVICAN60 dataset is partially annotated and the instances per class are very low [11]. LIVECell dataset, on the other hand, consists of 5,239 manually annotated, expertvalidated, phase contrast microscopy images with a total of $1,686,352$ individual cells annotated from eight different cell cultures. On average, the LIVECell dataset contains 313 cells per image which is very high as compared to the EVICAN dataset, which contains an average of 5.7 cells per image. That is the reason why we opted for the LIVECell dataset for this study. We have used two different variations of the LIVECell dataset.

\section{A. LIVECell8}

In this variation, instead of one generic cell class, we take each cell culture as a separate class. There is a total of eight classes in the LIVECell dataset. To avoid confusion, we have named the dataset in this research as LIVECell8. The microscopic images in this setting are monoculture i.e., every image contains cells from one cell culture only. Table I shows the distribution of different images and instances for each cell culture in train, validation, and test sets.

\section{B. Synthetic Co-culture images}

In this dataset variant, we have generated synthetic coculture images using the original LIVECell dataset as described in Section III. We have generated three different subsets for training namely DeepMuCS800, DeepMuCS1600, and DeepMuCS4000 each containing a different number of images and cell instances using the DeepMuCS-Generation. The information about the number of images and cell instances in different training subsets, validation, and test sets is mentioned in Table II.

\section{Evaluation Metrics}

To evaluate the performance of the proposed pipeline we are following the standard COCO evaluation protocol [6] with some modifications as reported in [4] for the area ranges.

Average Precision (AP) is the precision averaged across all unique recall levels. Mean Average Precision (mAP) is the mean of average precision across all $N$ classes. For the evaluation, we have reported mean average precision for both object detection and segmentation tasks at different IoU thresholds of 0.5 (mAP50), 0.75 (mAP75), and 0.5:0.95 in the steps of 0.05 (mAP). To identify the performance of the model on objects of different sizes, we have also included mAP for different area ranges. Objects with area less than $320 \mu^{2}$ (corresponding to 500 pixels) belong to APs (small). APm (medium) is for the objects in area ranges of $320 \mu^{2}$ to $970 \mu \mathrm{m}^{2}$ (corresponding to 1500 pixels) and APl (large) is for objects with area larger than $970 \mu \mathrm{m}^{2}$.

\section{EXPERIMENTAL SETUP}

DeepMuCS is evaluated in two different experimental settings. Different experimentation demonstrates the impact and limitations of multiple cell type segmentation in monoculture and co-culture microscopic images. In the first setting namely culture-dependent cell segmentation in monoculture, multiple cell type segmentation is performed on the monoculture microscopic images. The microscopic images used for this experiment contain a maximum of only one cell type per image. In the second experimental setting, different subsets of synthetic co-culture images for the training are used as described in Section V-B. The same validation (DeepMuCS_val) and test (DeepMuCS_test) set is used for the evaluation of all the different training subsets.

Training for all the experiments is performed with a base learning rate of 0.02 and momentum of 0.9 . The anchor sizes and aspect ratios for all settings were set after careful consideration of the cell's pixel area in the images. Anchor sizes and aspect ratios were set to $8,16,32,64,128$, and 0.5 , $1,2,3,4$ for all the settings respectively. The checkpoints for evaluation were chosen based on the lowest validation loss and higher validation average precision.

For image normalization, synchronized batch normalization [28] is used. The pixel means and pixel standard deviation for the dataset were calculated as 114.83, 114.83, 114.83, and $15.455,15.455,15.455$ respectively. For data augmentation, images are flipped horizontally on a random basis to reduce the risk of over-fitting. All training used multi-scale data augmentation, meaning that image sizes were randomly changed from the original $520 \times 704$ pixels to size with the same ratios, but the shortest side set to one of $(440,480,520,580,620)$ pixels. 
TABLE I: Summary statistics of images and cell instances in the LIVECell8 training, validation and test set.

\begin{tabular}{|c|c|c|c|c|c|c|c|c|}
\hline \multirow{2}{*}{ Cell type } & \multicolumn{2}{|c|}{ Total } & \multicolumn{2}{c|}{ Training } & \multicolumn{2}{c|}{ Validation } & \multicolumn{2}{c|}{ Test } \\
\cline { 2 - 9 } & Images & Cells & Images & Cells & Images & Cells & Images & Cells \\
\hline A172 & 608 & 131841 & 388 & 83175 & 68 & 15688 & 152 & 32978 \\
\hline BT-474 & 672 & 134542 & 429 & 85713 & 75 & 15088 & 168 & 33741 \\
\hline BV-2 & 608 & 383339 & 386 & 245638 & 70 & 45817 & 152 & 91884 \\
\hline Huh7 & 600 & 40882 & 352 & 24009 & 48 & 3886 & 200 & 12987 \\
\hline MCF7 & 735 & 353795 & 469 & 216052 & 82 & 39872 & 184 & 97871 \\
\hline SH-SY5Y & 704 & 274726 & 449 & 168791 & 79 & 28796 & 176 & 77139 \\
\hline SkBr3 & 704 & 264816 & 449 & 169366 & 79 & 29693 & 176 & 65757 \\
\hline SK-OV-3 & 608 & 102411 & 266 & 41363 & 38 & 5531 & 304 & 55517 \\
\hline Total & $\mathbf{5 2 3 9}$ & $\mathbf{1 6 8 6 3 5 2}$ & $\mathbf{3 1 8 8}$ & $\mathbf{1 0 3 4 1 0 7}$ & $\mathbf{5 3 9}$ & $\mathbf{1 8 4 3 7 1}$ & $\mathbf{1 5 1 2}$ & $\mathbf{4 6 7 8 7 4}$ \\
\hline
\end{tabular}

TABLE II: Summary statistics of images and cell instances in the synthetic co-culture training subsets, validation and test sets.

\begin{tabular}{|c|c|c|c|c|c|c|c|c|c|c|}
\hline \multirow[b]{2}{*}{ Dataset } & \multirow[b]{2}{*}{ Images } & \multirow[b]{2}{*}{ Total Cells } & \multicolumn{8}{|c|}{ Cells per culture } \\
\hline & & & A172 & В T-474 & BV-2 & Huh7 & MCF7 & SH-SY5Y & SkBr3 & SK-OV-3 \\
\hline DeерMuCS800 & 800 & 10137 & 341 & 982 & 759 & 125 & 1207 & 3630 & 2926 & 167 \\
\hline DeepMuCS1600 & 1600 & 19826 & 668 & 1998 & 1402 & 254 & 2347 & 6984 & 5808 & 365 \\
\hline DeepMuCS4000 & 4000 & 49613 & 1657 & 4732 & 3482 & 603 & 6031 & 17772 & 14438 & 898 \\
\hline DeepMuCS_val & 570 & 7120 & 227 & 667 & 523 & 101 & 875 & 2519 & 2092 & 116 \\
\hline DeepMuCS_test & 1564 & 19408 & 639 & 1843 & 1348 & 246 & 2388 & 6952 & 5668 & 324 \\
\hline
\end{tabular}

\section{A. Experimental Setting 1: Culture-dependent cell segmenta- tion in monoculture}

In this experimental setting, the objective is to detect, segment, and classify cells in monoculture microscopic images. There are eight different cell cultures in the LIVECell8 dataset. All the images in the dataset contain only one cell type per image. Table V-A gives insights into the data used for the training in this experimental setting.

The checkpoint at 5,000 iterations was chosen for evaluation for this experimental setting after observing the validation loss and the validation mean average precision.

1) Results: Table III shows the overall cell detection and segmentation results of DeepMuCS on the LIVECell8 dataset. Overall mean average precision (mAP) scores of $47.98 \%$ and $47.64 \%$ are achieved for detection and segmentation respectively.

Table IV shows the per class evaluation results for the culturedependent cell segmentation in monoculture environment. It can be observed that the best detection and segmentation performance is seen across cell culture $\mathrm{SkBr}$, followed by SK-OV-3 and BV-2. The worse performance can be seen across cell cultures SH-SY5Y followed by A172 and MCF7.

\section{B. Experimental Setting 2: Culture-dependent cell segmenta- tion in co-culture}

The purpose of this experimental setting is to analyze the performance of the proposed pipeline on the co-culture microscopic images. The synthetic dataset used in this setting contains images with multiple cell cultures in one image, which makes it a challenging task because unlike the instances in natural object dataset like MS-COCO [6] dataset, the cells in the phase-contrast microscopic images don't vary considerably. Most of the cell lines used to generate this synthetic co-culture dataset share similar morphological characteristics, which makes it difficult to distinguish between different cell cultures.

Table II give insights into generated synthetic data for training, validation and testing. Three different subsets were generated for training namely DeepMuCS800, DeepMuCS1600, and DeepMuCS4000. The same validation and test set is used to evaluate the performance of the models trained on different training subsets. Different cell culture instances distribution is also given in the Table II for each training subset, validation, and test set.

The checkpoints at 3,000, 6,500 and 6,500 iterations were chosen for the evaluation of DeepMuCS800, DeepMuCS1600, and DeepMuCS4000 respectively.

1) Results: Table III gives the overall detection and segmentation mAP scores averaged over all the eight-cell classes for the different training subsets. For the training subset DeepMuCS800, mAP scores of $70.17 \%$ and $69.31 \%$ are achieved for detection and segmentation respectively. The segmentation mAP scores for different area range for this setting show that the model performs very well for the cells of medium area range, followed by large and small area ranges. When we increase the images and cell instances for the training, the model performs nearly $7 \%$ better in terms of detection and segmentation mAP for the DeepMuCS1600 training subset. When we increase the data for training in DeepMuCS4000, the detection and segmentation scores further improve by around $1 \%$.

Table IV gives insights into per class evaluation results for the models trained on the three different synthetic co-culture training subsets. As we increase the training data, the performance across all the eight cell culture classes improves. For the DeepMuCS800, the best segmentation mAP score is achieved for the cell culture $\mathrm{SkBr} 3$, followed by A172. The worse performance in terms of segmentation $\mathrm{mAP}$ is seen for BV-2 followed by SH-SY5Y. The best performance in terms of segmentation mAP for the DeepMuCS1600 trained model is seen across the cell culture A172 followed by $\mathrm{SkBr} 3$ and the worse performance is recorded against the cell culture SHSY5Y. Similar to DeepMuCS1600, the best performance is seen for the cell culture A172 in terms of segmentation mAP, 
TABLE III: Overall detection and segmentation results on different Intersection over union threshold and area ranges for the two experimental settings

\begin{tabular}{|c|c|c|c|c|c|c|c|c|c|c|c|c|}
\hline \multicolumn{13}{|c|}{ Culture-dependent cell segmentation in monoculture } \\
\hline \multirow{2}{*}{ Train dataset } & \multicolumn{2}{|c|}{$\mathbf{m A P}$} & \multicolumn{2}{|c|}{ mAP50 } & \multicolumn{2}{|c|}{ mAP75 } & \multicolumn{2}{|c|}{ mAPs } & \multicolumn{2}{|c|}{$\mathbf{m A P m}$} & \multicolumn{2}{|c|}{ mAPI } \\
\hline & Det. & Seg. & Det. & Seg. & Det. & Seg. & Det. & Seg. & Det. & Seg. & Det. & Seg. \\
\hline LIVECell8 & 47.98 & 47.64 & 80.54 & 79.99 & 50.46 & 51.11 & 43.56 & 39.34 & 45.78 & 48.05 & 42.72 & 52.29 \\
\hline \multicolumn{13}{|c|}{ Culture-dependent cell segmentation in co-culture } \\
\hline DeepMuCS800 & 70.17 & 69.31 & 84.38 & 87.53 & 79.07 & 83.92 & 64.72 & 59.37 & 75.04 & 75.00 & 61.32 & 81.44 \\
\hline DeepMuCS1600 & 77.22 & 76.14 & 90.18 & 93.45 & 85.54 & 90.94 & 76.97 & 70.63 & 80.82 & 79.97 & 64.63 & 84.12 \\
\hline DeepMuCS4000 & 78.84 & 77.04 & 90.65 & 94.09 & 86.62 & 91.63 & 77.00 & 71.63 & 82.33 & 80.93 & 66.58 & 84.67 \\
\hline
\end{tabular}

TABLE IV: Per class average precision results for two experimental settings

\begin{tabular}{|c|c|c|c|c|c|c|c|c|c|c|c|c|c|c|c|c|}
\hline \multicolumn{17}{|c|}{ Culture-dependent cell segmentation in monoculture } \\
\hline Iram dataset & Det. & Seg. & Det. & Seg. & Det. & Seg. & Det. & Seg. & Det. & Seg. & Det. & Seg. & Det. & Seg. & Det. & Seg. \\
\hline LIV] & 38.48 & 39.99 & 44.31 & 44.03 & 56.88 & 54.90 & 54.08 & 53.20 & 39.48 & 39.75 & 30.20 & 27.39 & 65.710 & 66.52 & 54.71 & 55.31 \\
\hline \multicolumn{17}{|c|}{ Culture-dependent cell segmentation in co-culture } \\
\hline Deep & 79.18 & 82.14 & 76.65 & 75.46 & 74.79 & 72.66 & 78.66 & 77.17 & 79.73 & 75.45 & 76.82 & 68.61 & 80.52 & 81.01 & 71.42 & 76.62 \\
\hline DeepMuCS4000 & 79.84 & 83.24 & 77.21 & 75.45 & 76.91 & 74.46 & 78.11 & 75.85 & 81.90 & 76.58 & 79.53 & 70.63 & 82.47 & 82.31 & 74.97 & 77.83 \\
\hline
\end{tabular}

and the worst performance is detected for SH-SY5Y.

After examining the overall detection and segmentation mAP scores averaged over all the eight classes in Table III and the per class mAP scores in Table IV for the culture-dependent cell segmentation in co-culture environment, it is transparent that by increasing the images and cell instances per class the performance is improved.

\section{ANALYSIS AND DISCUSSION}

In this section, we discuss the results of DeepMuCS for both experimental settings. In experimental setting 1 (Culturedependent cell segmentation in monoculture), monoculture microscopic images containing a maximum of one cell culture per image are used for training and evaluation. DeepMuCS achieves good average precision scores for detection and segmentation tasks. For the segmentation task, we achieve an overall mAP score of $47.64 \%$ which is $0.25 \%$ less than what's reported in [4]. The difference in this experimental setting is that, in addition to detecting and segmenting cells, we are also concerned with classifying the cell culture for each cell in the microscopic images which is more challenging for deep learning algorithms. Analyzing the area ranges scores for the experimental setting 1 , the model finds it harder to segment cells of small areas as compared to medium and large area cells. LIVECell8 trained model performs best at detecting, segmenting, and classifying cell instances from the cultures $\mathrm{SkBr} 3$ followed by SK-OV-3, BV-2, and Huh7. The worse performance is seen across the cell culture SH-SY5Y because it belongs to the neuroblastoma cell line and neuronal cells have a unique morphology compared to the other cell types, tending to be highly asymmetric and concave-shaped due to their characteristic branching neuritis [4].

In the second experimental setting (Culture-dependent cell segmentation in co-culture), synthetic co-culture microscopic images with more than one cell culture per image are used for training and evaluation. Different training subsets with increasing images and cell instances per cell culture were used for training separate models using the proposed pipeline. The purpose of using different subsets is to analyze the segmentation and classification performance of the proposed pipeline with different scale variations in the dataset. The same validation and test set is used for the evaluation of the models trained on the multiple training subsets. It can be seen from the Table III and IV that by increasing the images and cell instances for each cell culture, the overall and per class segmentation performance increases. We achieve segmentation mAP scores of $69.31 \%, 76.14 \%$, and $77.04 \%$ for the training subsets DeepMuCS800, DeepMuCS1600, and DeepMuCS4000 respectively. The per-class performance scores also increase with increasing the data. The results for the experimental setting 2 manifest the potential of the proposed pipeline for cell type aware segmentation in a coculture environment in the microscopic images.

We have also performed the transferability analysis between the two experiments. The model trained on the LIVECell8 was tested on the co-culture test set and vice versa. The results show that the models trained on the co-culture dataset perform better on the LIVECell8 test set even though they are trained with almost $0.02 \%$ of the total cell instances in the LIVECell8. This shows that the deep learning model learns more diverse representations with multiple cell cultures in a single image than only one cell culture per image and the overall segmentation and classification performance also increases consequently.

Fig. 3 shows the inference results on some samples where our proposed pipeline performed adequately and inadequately for both the experimental settings. The first row shows the results for the first experimental setting and the last three rows represent the results for the second experimental setting. The left column shows adequate results, and the right column represents the inadequate results for the two experimental settings. The red, pink, blue, and green glow around each image depict the results for experimental setting 1, and the three variations (DeepMuCS800, DeepMuCS1600, and DeepMuCS400) of the experimental setting 2 respectively. The AP50 on top of every prediction sub-image represents the segmentation average precision score at IoU threshold of 0.5 . 


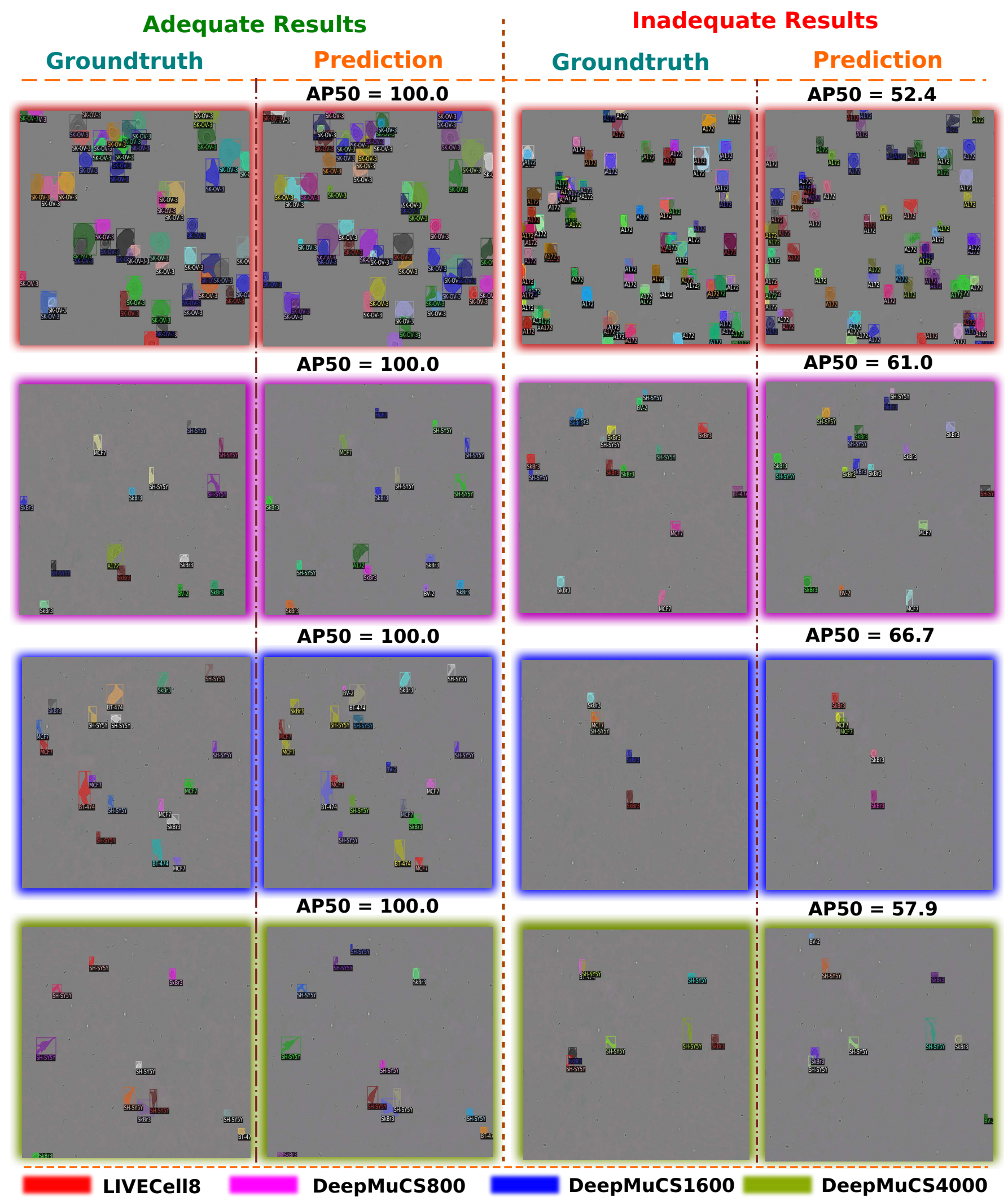

Fig. 3: Inference results of some samples where DeepMuCS-Segmentation performed adequately and inadequately for different experimental settings. The glow around the image represents the different experimental setting results. 
The predictions with detection scores above 0.5 are shown in the inference results.

For the culture-dependent cell segmentation in the monoculture experiment in the adequate column, we get an AP50 score of $100.0 \%$ with all the cell instances correctly segmented and classified i.e., SK-OV-3. The second row in the adequate column represents the results for the model trained on DeepMuCS800 for the culture-dependent cell segmentation experimental setting. The groundtruth of the image contains a total of 18 cell instances from four different cell cultures i.e., MCF-7, SkBr3, BT-474, and SH-SY5Y. All the cell instances are accurately detected, segmented, and classified according to their cell culture, hence the AP50 score of $100 \%$. In the third and the fourth row, we have adequate results for the DeepMuCS1600 and DeepMuCS4000. It can be observed that all instances are correctly predicted.

The first row in the inadequate column represent the results for the first experimental setting. AP50 score of 52.4\% is achieved for the test image. The groundtruth for this image contains 146 cell instances and LIVECell8 trained model missed a few cell instances and made some false positive predictions. In the second row, we have the results for the DeepMuCS800 trained model of the second experimental setting. The groundtruth for the image contains 16 cell instances from 4 different cell cultures. The model on a few occasions confused between the cell cultures SH-SY5Y and SkBr3, hence the AP50 score of $61.0 \%$. For the DeepMuCS1600 results, all the cell instances are correctly segmented by the model, but on one occurrence it confused SH-SY5Y cell instance for MCF7, hence the AP50 score of $66.7 \%$. The model made two false-positive predictions confusing background artifacts for the BV-2 cell culture and also missed one BT-474 cell instance resulting in an AP50 score of $57.9 \%$.

With the proposed approach and the experimental settings, we have demonstrated that it is possible to distinguish different cell cultures in the monoculture and co-culture microscopic images with good precision, which haven't been done before and can help the biologists to better understand the cellular mechanisms associated with disease states and also help in drug research.

\section{CONCLUSiOn}

In this study, we have presented a novel framework for multi-cell segmentation in monoculture and co-culture microscopic images. DeepMuCS provides a method to detect, segment, and classify each cell instance in the mono- and coculture microscopic images, which helps better quantification of individual cell appearance and behavior of different cell cultures. We have validated with the help of the proposed pipelines and different experimental settings that it is possible to distinguish different cell cultures in the microscopic images and achieve good performance. This study can help biologists to study the interaction between different cell populations and assist in drug research. The LIVECell dataset used in this study is composed of cells from different kinds of cancer. While our results demonstrate an impressive proof of concept for the method, cell type identification among different types of cancer cells is not biologically interesting as it would not be a situation that occurs naturally. The future direction for this work would be to validate the proposed workflow on a more clinically relevant co-culture model, like cancer cells mixed with stromal cells.

\section{REFERENCES}

[1] L. Axanova, D. J. Morré, and D. M. Morré, "Growth of lncap cells in monoculture and coculture with osteoblasts and response to tnox inhibitors," Cancer letters, 2005.

[2] A. P. Singh, S. Sharma, and D. K. Shah, "Quantitative characterization of in vitro bystander effect of antibody-drug conjugates," Journal of pharmacokinetics and pharmacodynamics, 2016.

[3] N. Khalid, M. Munir, C. Edlund, T. R. Jackson, J. Trygg, R. Sjögren, A. Dengel, and S. Ahmed, "Deepcens: An end-to-end pipeline for cell and nucleus segmentation in microscopic images," in 2021 International Joint Conference on Neural Networks (IJCNN). IEEE, 2021.

[4] C. Edlund, T. R. Jackson, N. Khalid, N. Bevan, T. Dale, A. Dengel, S. Ahmed, J. Trygg, and R. Sjögren, "Livecell—a large-scale dataset for label-free live cell segmentation," Nature methods, 2021.

[5] M. Schwendy, R. E. Unger, and S. H. Parekh, "Evican-a balanced dataset for algorithm development in cell and nucleus segmentation," Bioinformatics, 2020.

[6] T.-Y. Lin, M. Maire, S. Belongie, J. Hays, P. Perona, D. Ramanan, P. Dollár, and C. L. Zitnick, "Microsoft coco: Common objects in context," in European conference on computer vision. Springer, 2014.

[7] M. Hu, X. Ping, and Y. Ding, "Automated cell nucleus segmentation using improved snake," in 2004 International Conference on Image Processing, 2004. ICIP'04. IEEE, 2004.

[8] N. Lassouaoui and L. Hamami, "Genetic algorithms and multifractal segmentation of cervical cell images," in Seventh International Symposium on Signal Processing and Its Applications, 2003. Proceedings. IEEE, 2003

[9] T. Jiang and F. Yang, "An evolutionary tabu search for cell image segmentation," IEEE Transactions on Systems, Man, and Cybernetics, Part B (Cybernetics), 2002.

[10] R. Saha, M. Bajger, and G. Lee, "Spatial shape constrained fuzzy cmeans $(\mathrm{fcm})$ clustering for nucleus segmentation in pap smear images," in 2016 international conference on digital image computing: techniques and applications (DICTA). IEEE, 2016.

[11] N. Khalid, M. Munir, C. Edlund, T. R. Jackson, J. Trygg, R. Sjögren, A. Dengel, and S. Ahmed, "Deepcis: An end-to-end pipeline for celltype aware instance segmentation in microscopic images," in 2021 IEEE EMBS International Conference on Biomedical and Health Informatics (BHI). IEEE, 2021.

[12] J. W. Johnson, "Adapting mask-renn for automatic nucleus segmentation," arXiv preprint arXiv:1805.00500, 2018.

[13] Z. Cai and N. Vasconcelos, "Cascade r-cnn: Delving into high quality object detection," in Proceedings of the IEEE conference on computer vision and pattern recognition, 2018.

[14] Y. Song, L. Zhang, S. Chen, D. Ni, B. Li, Y. Zhou, B. Lei, and T. Wang, "A deep learning based framework for accurate segmentation of cervical cytoplasm and nuclei," in 2014 36th Annual International Conference of the IEEE Engineering in Medicine and Biology Society. IEEE, 2014.

[15] O. Ronneberger, P. Fischer, and T. Brox, "U-net: Convolutional networks for biomedical image segmentation," in International Conference on Medical image computing and computer-assisted intervention. Springer, 2015.

[16] D. A. Van Valen, T. Kudo, K. M. Lane, D. N. Macklin, N. T. Quach, M. M. DeFelice, I. Maayan, Y. Tanouchi, E. A. Ashley, and M. W. Covert, "Deep learning automates the quantitative analysis of individual cells in live-cell imaging experiments," PLoS computational biology, 2016.

[17] H.-F. Tsai, J. Gajda, T. F. Sloan, A. Rares, and A. Q. Shen, "Usiigaci: Instance-aware cell tracking in stain-free phase contrast microscopy enabled by machine learning," SoftwareX, 2019.

[18] E. Meijering, "Cell segmentation: 50 years down the road [life sciences]," IEEE signal processing magazine, 2012.

[19] T.-Y. Lin, P. Dollár, R. Girshick, K. He, B. Hariharan, and S. Belongie, "Feature pyramid networks for object detection," in Proceedings of the IEEE conference on computer vision and pattern recognition, 2017. 
[20] H. Zhang, C. Wu, Z. Zhang, Y. Zhu, Z. Zhang, H. Lin, Y. Sun, T. He, J. Mueller, R. Manmatha et al., "Resnest: Split-attention networks," arXiv preprint arXiv:2004.08955, 2020.

[21] J. Dai, H. Qi, Y. Xiong, Y. Li, G. Zhang, H. Hu, and Y. Wei, "Deformable convolutional networks," in Proceedings of the IEEE international conference on computer vision, 2017.

[22] K. He, X. Zhang, S. Ren, and J. Sun, "Deep residual learning for image recognition," in Proceedings of the IEEE conference on computer vision and pattern recognition, 2016.

[23] M. Zhang, X. Li, M. Xu, and Q. Li, "Automated semantic segmentation of red blood cells for sickle cell disease," IEEE Journal of Biomedical and Health Informatics, 2020.

[24] S. Ren, K. He, R. Girshick, and J. Sun, "Faster r-cnn: Towards realtime object detection with region proposal networks," arXiv preprint arXiv:1506.01497, 2015.

[25] K. He, G. Gkioxari, P. Dollár, and R. Girshick, "Mask r-cnn," in Proceedings of the IEEE international conference on computer vision, 2017.

[26] B. Roberts, A. Haupt, A. Tucker, T. Grancharova, J. Arakaki, M. A. Fuqua, A. Nelson, C. Hookway, S. A. Ludmann, I. A. Mueller et al., "Systematic gene tagging using crispr/cas9 in human stem cells to illuminate cell organization," Molecular biology of the cell, 2017.

[27] D. Gurari, D. Theriault, M. Sameki, B. Isenberg, T. A. Pham, A. Purwada, P. Solski, M. Walker, C. Zhang, J. Y. Wong et al., "How to collect segmentations for biomedical images? a benchmark evaluating the performance of experts, crowdsourced non-experts, and algorithms," in 2015 IEEE winter conference on applications of computer vision. IEEE, 2015

[28] H. Zhang, K. Dana, J. Shi, Z. Zhang, X. Wang, A. Tyagi, and A. Agrawal, "Context encoding for semantic segmentation," in Proceedings of the IEEE conference on Computer Vision and Pattern Recognition, 2018. 